Louisiana State University

LSU Digital Commons

Faculty Publications

Department of Biological Sciences

3-1-2017

\title{
Slow Equilibration between Spectroscopically Distinct Trap States in Reduced $\mathrm{TiO}_{2}$ Nanoparticles
}

\author{
Jennifer L. Peper \\ Yale University \\ David J. Vinyard \\ Yale University \\ Gary W. Brudvig \\ Yale University \\ James M. Mayer \\ Yale University
}

Follow this and additional works at: https://digitalcommons.Isu.edu/biosci_pubs

\section{Recommended Citation}

Peper, J., Vinyard, D., Brudvig, G., \& Mayer, J. (2017). Slow Equilibration between Spectroscopically Distinct Trap States in Reduced $\mathrm{TiO}_{2}$ Nanoparticles. Journal of the American Chemical Society, 139 (8), 2868-2871. https://doi.org/10.1021/jacs.6b12112

This Article is brought to you for free and open access by the Department of Biological Sciences at LSU Digital Commons. It has been accepted for inclusion in Faculty Publications by an authorized administrator of LSU Digital Commons. For more information, please contact ir@lsu.edu. 


\title{
Slow Equilibration between Spectroscopically Distinct Trap States in Reduced $\mathrm{TiO}_{2}$ Nanoparticles
}

\author{
Jennifer L. Peper, ${ }^{\circledR}$ David J. Vinyard, Gary W. Brudvig, ${ }^{\circledR}$ and James M. Mayer* \\ Department of Chemistry, Yale University, New Haven, Connecticut 06520-8107, United States
}

\section{Supporting Information}

ABSTRACT: Understanding the nature of charge carriers in nanoscale titanium dioxide is important for its use in solar energy conversion, photocatalysis, and other applications. UV-irradiation of aqueous, colloidal $\mathrm{TiO}_{2}$ nanoparticles in the presence of methanol gives highly reduced suspensions. Two distinct types of electron traps were observed and characterized by EPR and optical spectroscopies. The relative populations of the states depend on temperature, indicating a small energy difference, $\Delta H^{\circ}=3.0 \pm 0.6 \mathrm{kcal} / \mathrm{mol}(130 \pm 30 \mathrm{meV})$. Interconversion between the electron traps occurs slowly over the course of minutes to hours within the temperature range studied here, $0-50{ }^{\circ} \mathrm{C}$. The slow time scale implies that interconversion involves changes in structure or stoichiometry, not just the movement of electrons. This occurrence of slow structural modification with changes in trap state occupancy is likely a general feature of reduced $\mathrm{TiO}_{2}$ systems at thermodynamic equilibria or photostationary states and should be considered in the design of $\mathrm{TiO}_{2}$-containing devices.

$I^{\text {ntents }}$ nterest in titanium dioxide and other abundant metal oxide semiconductors has grown dramatically in recent decades as these materials take a prominent role in future photocatalytic and solar energy conversion technologies. ${ }^{1}$ Many studies have shown the diverse redox reactivity of $\mathrm{TiO}_{2}$ via electrons and holes generated chemically, photochemically, or electrochemically. $^{1-3}$ Spectroscopic studies have helped to elucidate the complex electronic landscape of reduced $\mathrm{TiO}_{2}$ systems $\left(\mathrm{TiO}_{2} /\right.$ $\left.e^{-}\right)$, which includes conduction band occupancy and intraband gap trapping of $e^{-}$at $\mathrm{Ti}$ (III)-based surface and interstitial sites. $^{2,4-7}$ The trap states are sensitive to solvent, $\mathrm{pH}$, surface ligands, and other parameters, but they remain poorly characterized. $^{7,8}$ The interconversions of electrons between different states have been identified as key steps in productive and nonproductive pathways for charge transport in $\mathrm{TiO}_{2}$ dye sensitized solar cells and other devices. ${ }^{9-11}$ Although transient studies of $\mathrm{TiO}_{2}$ under UV-photolysis have revealed important short time scale processes, i.e. electron trapping, connecting these results with the steady-state operation of $\mathrm{TiO}_{2}$ devices is difficult. $^{12}$ Bringing these effects together into a unified understanding of reactive extra electrons is crucial to the implementation of $\mathrm{TiO}_{2}$ into future technologies.

Herein we present studies of trapped electrons in welldefined aqueous uncapped colloidal $\mathrm{TiO}_{2}$ nanoparticles (NPs). Unlike most prior studies of transient or photostationary states, the long-term stability of the reduced $\mathrm{NPs}\left(\mathrm{TiO}_{2} / e^{-}\right)$studied here allowed for the trap states to reach their thermodynamic equilibrium. Two distinct classes of electronic states were observed. By correlating the electron paramagnetic resonance (EPR) and UV-vis-NIR spectra of $\mathrm{TiO}_{2} / e^{-}$, the ill-defined, broad visible feature of the reduced NPs was deconvoluted and extinction coefficients of each type of electronic state were determined. Thermal equilibration studies showed the energy difference between the two types of electrons to be small. Surprisingly, equilibration of the two sites occurs very slowly (minutes to hours), indicating that equilibration involves changes in structure or composition rather than just electronic reorganization. Although the detailed behavior of these states may be specific to the $\mathrm{TiO}_{2}$ nanoparticle system studied here, the general conclusions are likely applicable to most reduced $\mathrm{TiO}_{2}$ systems.

Anatase titanium dioxide NPs with a diameter of $4 \pm 1 \mathrm{~nm}$ were synthesized via hydrolysis of $\mathrm{TiCl}_{4}$ as previously described. ${ }^{13,14}$ The resulting white solid was resuspended in neutral $18 \mathrm{M} \Omega \mathrm{cm}$ water to give a transparent colloid with $\mathrm{pH}$ 2.1-2.3. Freshly suspended $\mathrm{TiO}_{2} \mathrm{NPs}$ were reduced by UVirradiation of the colloid in the presence of $0.1 \mathrm{M}$ methanol under an inert atmosphere at room tempurature. ${ }^{13}$ A blue-violet coloration and a broad optical feature appeared during photolysis. The reduced colloid was essentially stable for several days under an inert atmosphere. Upon exposure to air, $\mathrm{TiO}_{2} / e^{-}$quickly oxidized.

The total electron concentration in $\mathrm{TiO}_{2} / e^{-}$colloids was determined via titrations with 4-MeO-TEMPO (4-methoxy2,2,6,6-tetramethyl-1-piperidinyloxy) (Figure 1A). ${ }^{15}$ Beer's Law plots, monitoring $\mathrm{TiO}_{2} / e^{-}$oxidation at $600 \mathrm{~nm}$, gave the molar extinction coefficient describing the electron concentration in the colloid ( $\mathrm{mol} e^{-} \mathrm{L}^{-1}$ ) as $230 \pm 35 \mathrm{M}^{-1} \mathrm{~cm}^{-1}$ at rt (Figure S3B). Colloids irradiated for $\sim 4$ h typically had $1.5 \pm 0.2 \mathrm{mM}$ $e^{-}$which corresponds to an average of $40-60 e^{-} / \mathrm{NP}\left(\sim 10^{21}\right.$ $\left.e^{-} \mathrm{cm}^{-3}\right) .^{14}$

EPR measurements of frozen $\mathrm{TiO}_{2} / e^{-}$samples revealed complex spectra. The spectra were simulated as the sum of two components, a rhombic signal $(g=1.990,1.898,1.875)$ and a broad axial signal $(g=1.937,1.850)$, indicating two distinct types of environments for the added electrons (Figure 1B, Supporting Information (SI) Section 3.1). ${ }^{16}$ Prior studies on similar $\mathrm{TiO}_{2}$ NPs have reported two (or more) trap environments and have concluded that $g<2$ values are consistent with $\mathrm{Ti}(\mathrm{III})$-based electrons. ${ }^{6,7}$

Received: November 23, 2016

Published: February 8, 2017 

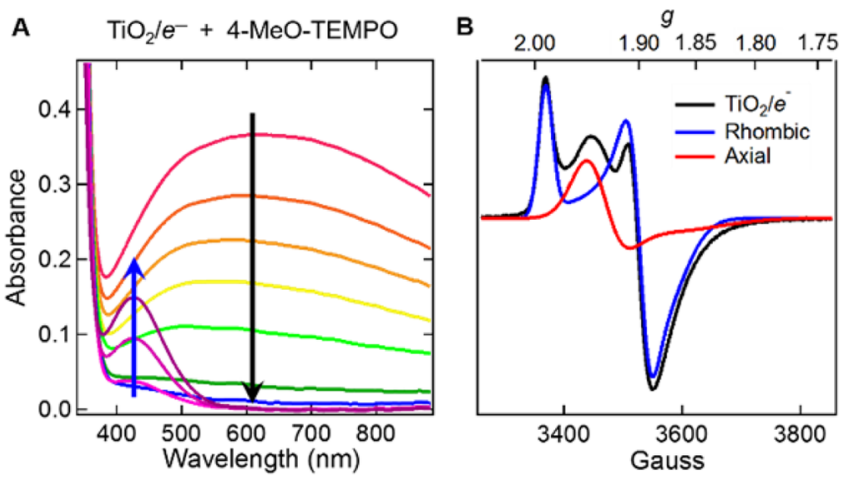

Figure 1. (A) Optical spectra of a $\mathrm{TiO}_{2} / e^{-}$titration with 4-MeOTEMPO. Consumption of $\mathrm{TiO}_{2} / e^{-}$(black arrow) is followed by the appearance of the oxidant (blue arrow). (B) EPR spectrum of the $\mathrm{TiO}_{2} / e^{-}$colloid at $9 \mathrm{~K}$ (black), simulated with a rhombic (blue) and an axial (red) signal.

During optical studies of the $\mathrm{TiO}_{2} / e^{-}$colloids it became evident that careful control of the sample temperature was required to obtain reproducible results. To probe the effect of temperature, aliquots of a $\mathrm{TiO}_{2} / e^{-}$colloid were equilibrated to $0,21.5$, and $35^{\circ} \mathrm{C}$ for at least $1 \mathrm{~h}$ before spectra were collected in a temperature controlled cuvette holder. Surprisingly, the $\mathrm{TiO}_{2} / e^{-}$coloration increased dramatically with sample temperature across the entire visible spectrum, with a $50 \%$ increase in absorbance density from 0 to $35{ }^{\circ} \mathrm{C}$ (Figure $2 \mathrm{~A}$ ). Changes in
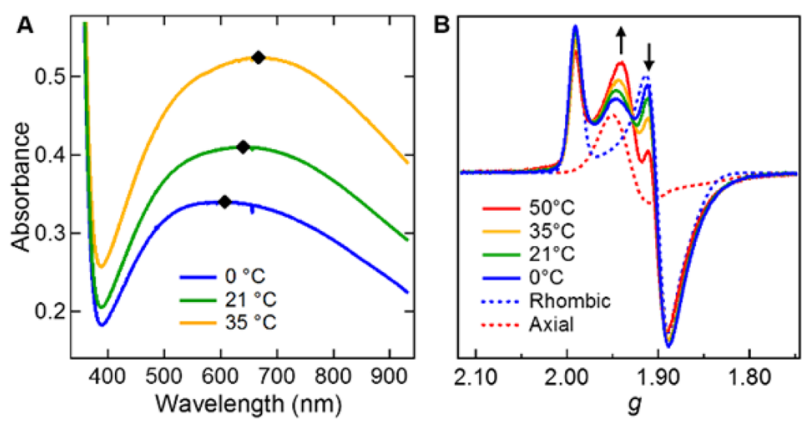

Figure 2. (A) Optical spectra of aliquots of the same $\mathrm{TiO}_{2} / e^{-}$sample after equilibration to different temperatures. Black markers indicate $\lambda_{\text {max }}$ (B) EPR spectra (normalized to spin density) of $\mathrm{TiO}_{2} / e^{-}$preequilibrated to different temperatures prior to rapid freezing. Dashed lines show the axial and rhombic components of the $21^{\circ} \mathrm{C}$ sample. Arrows denote trends with increased temperature.

absorbance were most prominent at longer wavelengths resulting in a subtle redshift in $\lambda_{\max }$ upon heating. Reequilibration of all $\mathrm{TiO}_{2} / e^{-}$aliquots to $\mathrm{rt}$ resulted in nearly identical UV-vis spectra indicating the full reversibility of the thermal process (Figure S9). Analysis of the colloid by dynamic light scattering (DLS) showed only small changes in the extent of NP aggregation as a function of temperature (SI Section 2.4). The changes in the $\mathrm{TiO}_{2} / e^{-}$visible peak with temperature imply a shift in the nature of the additional electrons in the reduced nanoparticles.

The temperature sensitivity of the electronic environments in $\mathrm{TiO}_{2} / e^{-}$was confirmed by corresponding EPR measurements. Temperature-equilibrated $\mathrm{TiO}_{2} / e^{-}$samples at $0-50{ }^{\circ} \mathrm{C}$ were quickly cooled to $77 \mathrm{~K}$ within $25 \mathrm{~s}$ before EPR measurements at $9 \mathrm{~K}$. Like the optical data, the EPR spectra shift dramatically as a function of the pre-equilibrated sample temperature (Figure
2B). All spectra are well fit by differing ratios of the rhombic and axial simulation components, with spin density shifting to the axial component as $T$ increases (Table S2). EPR measurements after re-equlibration of the $\mathrm{TiO}_{2} / e^{-}$samples to $22{ }^{\circ} \mathrm{C}$ for at least $1.5 \mathrm{~h}$ showed the convergence of all spectra to the $22{ }^{\circ} \mathrm{C}$ sample, except for the sample heated to $50{ }^{\circ} \mathrm{C}$, which did not fully re-equilibrate on this time scale (Figure S8, Table S2). These results and additional experiments (SI Section 3.2) show that the process of freezing the samples to obtain EPR spectra does not affect the electronic equilibrium between the two components. Therefore, the EPR spectra are accurate representations of $\mathrm{TiO}_{2} / e^{-}$at the pre-equilibrated temperatures and confirm the shift of electron density between electronic environments upon temperature modulation.

Based on the two-component fit of the EPR spectra, the optical spectra were quantitatively fit using two broad Gaussian peaks (Figure 3A). The occupied electronic states correspond-
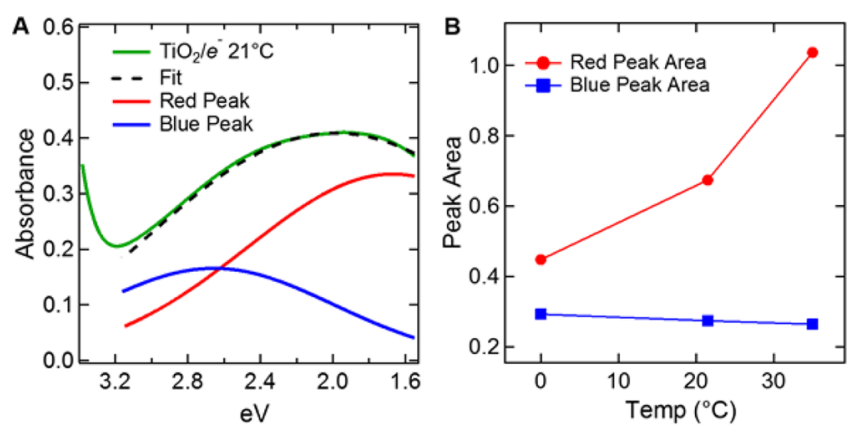

Figure 3. (A) The spectrum of $\mathrm{TiO}_{2} / e^{-}$at $21{ }^{\circ} \mathrm{C}$ plotted vs $\mathrm{eV}$ (green) and the optical fit (black, dashed) composed of two broad Gaussians (red and blue). (B) Red $/ e^{-}$and Blue $/ e^{-}$peak areas plotted as a function of the equilibration temperature.

ing to the peaks at $1.67 \mathrm{eV}(740 \mathrm{~nm})$ and $2.66 \mathrm{eV}(470 \mathrm{~nm})$ were named red $\left(\operatorname{Red} / e^{-}\right)$and blue $\left(\right.$Blue $/ e^{-}$) electrons, respectively, based on their respective $\lambda_{\max }$ values. $\mathrm{TiO}_{2} / e^{-}$ spectra at all three temperatures were fit with these fixed peak positions while the peak height and width were allowed to vary. Substantial broadening of the peaks at higher temperatures was observed, so comparisons were made using peak areas rather than peak heights (Figure 3B). Several other paired Gaussian peak fits were explored, and three additional models that adequately fit the spectra are presented in SI Section 4.3. All of the successful two-component fits are characterized by a highenergy peak (Blue $/ e^{-}$) and a more dominant, low-energy peak $\left(\operatorname{Red} / e^{-}\right)$, and all lead to the same conclusions.

At all temperatures, the low energy $\operatorname{Red} / e^{-}$component dominates the optical spectrum. Upon increasing the temperature from 0 to $35{ }^{\circ} \mathrm{C}$, the area of the red peak more than doubled while the Blue $/ e^{-}$peak decreased by only $\sim 10 \%$ (Figure $3 \mathrm{~B}$ ). The greater temperature sensitivity of $\operatorname{Red} / e^{-}$is illustrated graphically in Figure $3 \mathrm{~B}$ by the steeper temperature dependence of the $\operatorname{Red} / e^{-}$peak area. Assuming mass balance between the two species, the ratio of these dependencies (Figure 3B slopes) is equal to the ratio of the $\operatorname{Red} / e^{-}$and Blue $/ e^{-}$oscillator strengths, $f_{\operatorname{Red} / e^{-} /} / f_{\text {Blue } / e^{-}}=20 \pm 6 .{ }^{14}$ This rough calculation shows that the molar absorptivity of Red $/ e^{-}$ is much larger than that of Blue $/ e^{-}$.

The parallel temperature dependences of the two spectroscopic data sets show the correlation of the blue optical peak with the rhombic EPR signal (Blue $/ e^{-}$) and the red peak with the axial signal $\left(\operatorname{Red} / e^{-}\right)$(Figure 4$)$. This is the first report of 

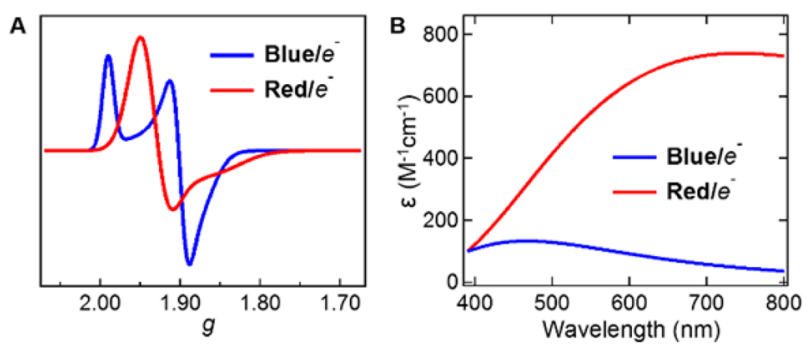

Figure 4. Rhombic and axial EPR components (A) and the molar absorptivity of the visible peaks (B) for Red $/ e^{-}$and Blue $/ e^{-}$.

substantial and coinciding temperature dependences of reduced $\mathrm{TiO}_{2}$ EPR and optical spectroscopies. The data show that the Blue $/ e^{-}$and $\mathbf{R e d} / e^{-}$are distinct classes of electron states, whose differences are much larger than the distributions within each class.

The correlation of these two data sets allows for the determination of the molar absorptivity of Red $/ e^{-}$and Blue $/ e^{-}$ (Figure $4 \mathrm{~B})$. The fraction of total electrons $\left(\left[e^{-}\right]_{\text {tot }}\right)$ contributing to the Blue $/ e^{-}$and Red $/ e^{-}$visible peaks is equal to the fraction of the rhombic and axial EPR spin densities. The smaller blue peak in Figure 3A represents $73 \%$ of $\left[e^{-}\right]_{\text {tot }}$; thus, Blue $/ e^{-}$has a much lower molar absorptivity than $\operatorname{Red} / e^{-}$ across the entire visible spectrum. ${ }^{14}$ The Blue $/ e^{-}$absorption band $\left(\varepsilon_{470 \mathrm{~nm}}=130 \mathrm{M}^{-1} \mathrm{~cm}^{-1}\right)$ is similar to $\mathrm{d}-\mathrm{d}$ optical transitions observed at other $\mathrm{Ti}^{3+}$ ions with the somewhat higher intensity consistent with the distortion of $\mathrm{Ti}$ centers within an anatase lattice. ${ }^{17}$ The breadth, high absorptivity, and location of the Red $/ e^{-}$peak $\left(\varepsilon_{740 \mathrm{~nm}}=740 \mathrm{M}^{-1} \mathrm{~cm}^{-1}\right)$ are consistent with previous assignments of intervalence charge transfer bands within $\mathrm{Ti}^{3+}$-containing minerals. ${ }^{18}$ These titanium-based peak assignments and the corresponding $g$ values are consistent with isolated electronic trap states.

$$
\text { Blue } / \mathrm{e}^{-}+\operatorname{Red}^{\circ} \rightleftharpoons \text { Blue }^{\circ}+\operatorname{Red} / \mathrm{e}^{-}
$$

The EPR and optical data demonstrate substantial and reversible shifts of $e^{-}$from Blue $/ e^{-}$to Red $/ e^{-}$electronic states with small increases in temperature. The simple electrontransfer model for this equilibrium in eq 1 uses $\left[\right.$ Blue $\left./ e^{-}\right]$, for instance, to be the concentration of electrons in blue states $\left(\mathrm{mol}\right.$ "Blue $\left./ e^{-} \mathrm{L}^{-1}\right)$ and $\left[\mathrm{Blue}^{\circ}\right]$ as the concentration of unreduced blue states. The sum of $\left[\right.$ Blue $\left./ e^{-}\right]$and $\left[\operatorname{Red} / e^{-}\right]$is determined by titration with 4-MeO-TEMPO, and their ratio was measured by the integrations of their respective components in EPR spectra. Although the concentrations of unreduced states, $\left[\operatorname{Red}^{\circ}\right]$ and $\left[\mathrm{Blue}^{\circ}\right]$, are unknown, they are likely much larger than their reduced forms, as indicated by the constant ratio of Red $/ e^{-}$to Blue $/ e^{-}$as a function of $\mathrm{TiO}_{2}$ reduction (SI Section 3.3). With this assumption, $\left[\mathrm{Blue}^{\circ}\right] /$ $\left[\operatorname{Red}^{\circ}\right]$ is a constant $(C)$, and $K_{\mathrm{eq}}=C\left[\operatorname{Red} / e^{-}\right] /\left[\right.$Blue $\left./ e^{-}\right]$. A van't Hoff analysis then gives an enthalpic difference between Blue $/ e^{-}$and Red $/ e^{-}$of $3.0 \pm 0.6 \mathrm{kcal} / \mathrm{mol}(0.13 \pm 0.03 \mathrm{eV})$, independent of the value of $C$ (Figure 5a). The small thermodynamic preference for the Blue/ $e^{-}$states is consistent with the transfer of approximately $20 \%$ of the total electrons from blue to red states upon heating from 0 to $50{ }^{\circ} \mathrm{C}$.

All of the results reported here can be understood with a simplified model showing Red $/ e^{-}$and Blue $/ e^{-}$as distinct intraband gap trap states separated by $0.13 \mathrm{eV}$ (Figure 5B). Similar multistate models have been proposed previously based on photoluminescence spectra of reduced $\mathrm{TiO}_{2}{ }^{2,5}$ While only a
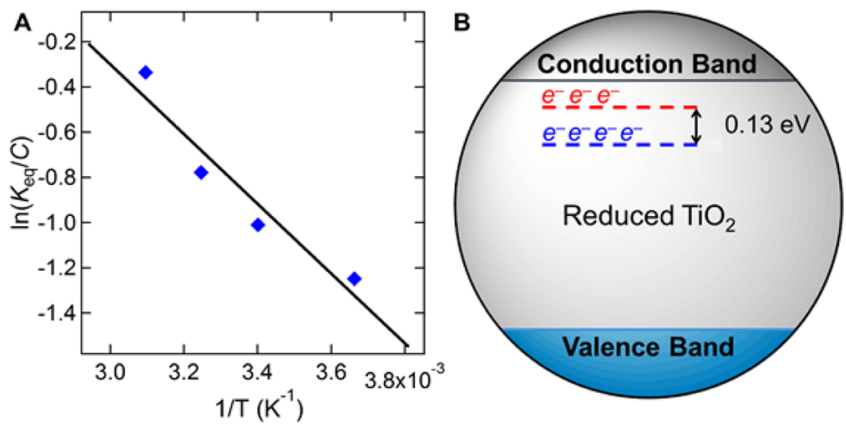

Figure 5. (A) van't Hoff plot of $K_{\mathrm{eq}} / C$ determined from the ratio of the $\mathrm{Red} / e^{-}$and Blue $/ e^{-}$EPR components of $\mathrm{TiO}_{2} / e^{-}$samples preequilibrated to $0-50{ }^{\circ} \mathrm{C}$. (B) A simplified band diagram showing Red/ $e^{-}$and Blue $/ e^{-}$as intraband gap states.

few $e^{-}$are indicated in the figure, each charged nanoparticle contains $40-60 e^{-}$(ca. 5\% of Ti ions are reduced).

Surprisingly, the equilibration of $\mathrm{TiO}_{2} / e^{-}$at different temperatures is very slow. All the experiments showed the optical changes lagging several minutes to hours behind changes in sample temperature. For instance, a $35{ }^{\circ} \mathrm{C} \mathrm{TiO}_{2} /$ $e^{-}$sample was cooled to $21{ }^{\circ} \mathrm{C}$ in less than $10 \mathrm{~min}$; however, the $\mathrm{TiO}_{2} / e^{-}$absorbance required $7 \mathrm{~h}$ for full equilibration (Figure 6). The relative absorbance change in Figure 6 is

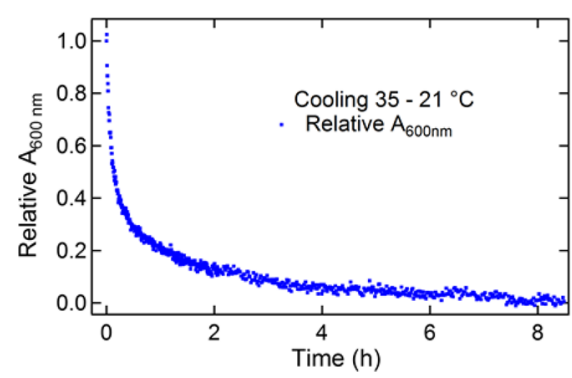

Figure 6. Change in the relative $A_{600 \mathrm{~nm}}$ of $\mathrm{TiO}_{2} / e^{-}$over time upon cooling from $35-21{ }^{\circ} \mathrm{C}$.

nonexponential, and the first $75 \%$ occurs within $45 \mathrm{~min}$. The equilibrations of $\mathrm{TiO}_{2} / e^{-}$to warmer temperatures were faster, generally completing within 20 min (Figure S10).

The minutes/hours time scale of the spectroscopic changes observed here are much slower than the processes ascribed to electron trapping/detrapping observed in photochemical experiments with $\mathrm{TiO}_{2}{ }^{.2}$ Instead, this long time scale is reminiscent of the electrochemical reduction of $\mathrm{TiO}_{2}$ films, often attributed to $\mathrm{Li}^{+}$or $\mathrm{H}^{+}$intercalation. ${ }^{11,19}$ In our study, however, the slow equilibration is for isomerization of electrons between trap states without any change in the extent of $\mathrm{TiO}_{2}$ reduction. The isomerization, being too slow for simple electron transfer, indicates that structural changes at the Red/ $e^{-}$and Blue $/ e^{-}$trap states accompany the movement of electrons. These changes are likely movement(s) of protons and/or oxide/hydroxide anions. ${ }^{3}$ Some prior studies of $\mathrm{TiO}_{2}$ devices have ascribed slow charge transport to the formation of proton-stabilized electron trap states. ${ }^{10,11}$

In conclusion, parallel optical and EPR studies of reduced aqueous $\mathrm{TiO}_{2}$ nanoparticles show the presence of two stable and spectroscopically distinct classes of electronic environments, termed Red $/ e^{-}$and Blue $/ e^{-}$, each of which encompasses a group of states with similar energy and 
symmetry. Temperature modulation over a narrow range, $0-50$ ${ }^{\circ} \mathrm{C}$, showed substantial and reversible shifts in the optical and EPR spectra of $\mathrm{TiO}_{2} / e^{-}$. This is the first study to demonstrate a clear correlation between the EPR and optical signals of reduced $\mathrm{TiO}_{2}$. The tandem use of the two spectroscopies, combined with the stability of the system, allowed for an unprecedented quantitative analysis of the ill-defined, broad $\mathrm{TiO}_{2} / e^{-}$optical signal. The greater molar absorptivity of Red/ $e^{-}$states relative to $\mathrm{Blue} / e^{-}$is the root of the dramatic temperature sensitivity of the $\mathrm{TiO}_{2} / e^{-}$visible signal and is likely a contributing factor to the large range of previously reported epsilons for similar $\mathrm{TiO}_{2} / e^{-}$systems. ${ }^{13,20}$

Quantitative van't Hoff analysis of the equilibrium between the classes of states shows that the Blue/ $e^{-}$are $\sim 130 \mathrm{meV}$ more stable than the $\operatorname{Red} / e^{-}$. This model of energetically distinct classes of trap states in $\mathrm{TiO}_{2} / e^{-}$has been previously suggested but is less common than the alternative model of a broad energetic distribution of trap states in semiconductors. $^{2,5,21}$ Remarkably, equilibration between the $\operatorname{Red} / e^{-}$and Blue $/ e^{-}$states is established very slowly, over minutes to hours. It has, therefore, not been observed in the common transient optical experiments. The slow equilibration indicates that interconversion involves structural and/or stoichiometric changes in the trap states, not solely electron transfer.

While the particulars of the Red $/ e^{-}$and Blue $/ e^{-}$sites seem to be specific to these aqueous colloidal nanoparticles, the principles are likely to be general for $\mathrm{TiO}_{2}$ and many other materials in contact with protic media: slow equilibration of chemically distinct trap states, with structural and/or compositional change, in response to changes in temperature (or other stimuli). These slow rearrangements between electronic states are likely important in steady-state device operation, which may involve states quite different than those observed in short time scale photophysical experiments.

\section{ASSOCIATED CONTENT}

\section{S Supporting Information}

The Supporting Information is available free of charge on the ACS Publications website at DOI: 10.1021/jacs.6b12112.

Experimental procedures and analytical methods (PDF)

\section{AUTHOR INFORMATION}

\section{Corresponding Author}

*E-mail: james.mayer@yale.edu.

\section{ORCID 우}

Jennifer L. Peper: 0000-0002-2611-1011

Gary W. Brudvig: 0000-0002-7040-1892

Notes

The authors declare no competing financial interest.

\section{ACKNOWLEDGMENTS}

This work was supported as part of the Argonne-Northwestern Solar Energy Research (ANSER) Center, an Energy Frontier Research Center funded by the U.S. Department of Energy, Office of Science, Basic Energy Sciences under Award No. DESC0001059. DLS measurements were performed in the Facility for Light Scattering at Yale University.

\section{REFERENCES}

(1) Ma, Y.; Wang, X.; Jia, Y.; Chen, X.; Han, H.; Li, C. Chem. Rev. 2014, 114, 9987.
(2) Rex, R. E.; Knorr, F. J.; McHale, J. L. J. Phys. Chem. C 2014, 118, 16831.

(3) Wheeler, D. A.; Ling, Y.; Dillon, R. J.; Fitzmorris, R. C.; Dudzik, C. G.; Zavodivker, L.; Rajh, T.; Dimitrijevic, N. M.; Millhauser, G.; Bardeen, C.; Li, Y.; Zhang, J. Z. J. Phys. Chem. C 2013, 117, 26821.

(4) Berger, T.; Sterrer, M.; Diwald, O.; Knözinger, E.; Panayotov, D.; Thompson, T. L.; Yates, J. T. J. Phys. Chem. B 2005, 109, 6061.

(5) Ghosh, A. K.; Wakim, F. G.; Addiss, R. R. Phys. Rev. 1969, 184, 979.

(6) Howe, R. F.; Graetzel, M. J. Phys. Chem. 1985, 89, 4495.

(7) Rajh, T.; Nedeljkovic, J. M.; Chen, L. X.; Poluektov, O.; Thurnauer, M. C. J. Phys. Chem. B 1999, 103, 3515.

(8) Redmond, G.; Fitzmaurice, D.; Graetzel, M. J. Phys. Chem. 1993, 97, 6951.

(9) Konezny, S. J.; Richter, C.; Snoeberger, R. C.; Parent, A. R.; Brudvig, G. W.; Schmuttenmaer, C. A.; Batista, V. S. J. Phys. Chem. Lett. 2011, 2, 1931.

(10) Halverson, A. F.; Zhu, K.; Erslev, P. T.; Kim, J. Y.; Neale, N. R.; Frank, A. J. Nano Lett. 2012, 12, 2112.

(11) Swierk, J. R.; McCool, N. S.; Saunders, T. P.; Barber, G. D.; Mallouk, T. E. J. Am. Chem. Soc. 2014, 136, 10974.

(12) Schneider, J.; Matsuoka, M.; Takeuchi, M.; Zhang, J.; Horiuchi, Y.; Anpo, M.; Bahnemann, D. W. Chem. Rev. 2014, 114, 9919.

(13) Mohamed, H. H.; Mendive, C. B.; Dillert, R.; Bahnemann, D. W. J. Phys. Chem. A 2011, 115, 2139.

(14) See Supporting Information.

(15) Schrauben, J. N.; Hayoun, R.; Valdez, C. N.; Braten, M.; Fridley, L.; Mayer, J. M. Science 2012, 336, 1298.

(16) Stoll, S.; Schweiger, A. J. Magn. Reson. 2006, 178, 42.

(17) Figgis, B. N.; Hitchman, M. A. Ligand Field Theory and Its Applications; King, R. B., Ed.; Wiley-VCH: New York, 2000.

(18) Khomenko, V. M.; Langer, K.; Rager, H.; Fett, A. Phys. Chem. Miner. 1998, 25, 338.

(19) Song, W.; Luo, H.; Hanson, K.; Concepcion, J. J.; Brennaman, M. K.; Meyer, T. J. Energy Environ. Sci. 2013, 6, 1240.

(20) Koelle, U.; Moser, J.; Graetzel, M. Inorg. Chem. 1985, 24, 2253.

(21) Bai, Y.; Mora-Seró, I.; De Angelis, F.; Bisquert, J.; Wang, P. Chem. Rev. 2014, 114, 10095. 